\title{
A test of cosmic distance duality relation using SPT-SZ galaxy clusters, Type Ia supernovae, and cosmic chronometers
}

\author{
Kamal Bora* and Shantanu Desai ${ }^{\dagger}$ \\ Department of Physics, Indian Institute of Technology, Hyderabad, Kandi, Telangana-502285, India
}

(Dated: June 10, 2021)

\begin{abstract}
We carry out a test of the cosmic distance duality relation using a sample of 52 SPT-SZ clusters, along with X-ray measurements from XMM-Newton. To carry out this test, we need an estimate of the luminosity distance $\left(D_{L}\right)$ at the redshift of the cluster. For this purpose, we use three independent methods: directly using $D_{L}$ from the closest Type Ia Supernovae from the Union 2.1 sample, non-parametric reconstruction of $D_{L}$ using the same Union 2.1 sample, and finally using $H(z)$ measurements from cosmic chronometers and reconstructing $D_{L}$ using Gaussian Process regression. We use four different functions to characterize the deviations from CDDR. All our results for these $(4 \times 3)$ analyses are consistent with CDDR to within $1 \sigma$.
\end{abstract}

\section{INTRODUCTION}

One of the most fundamental tenet in Cosmology is the cosmic distance duality relation (CDDR, hereafter) between the angular diameter distance $\left(D_{A}\right)$ and the luminosity distance $\left(D_{L}\right)$, which is sometimes also known as the Etherington relation $[1,2]$. This relation is given by:

$$
\eta(z) \equiv \frac{D_{L}}{D_{A}(1+z)^{2}}=1
$$

The CDDR involves three assumptions [3, 4]: spacetime is endowed with a metric theory of gravity; photons propagate along null geodesics; and the total number of photons is conserved. The CDDR relation is one of the edifice of the standard $\Lambda \mathrm{CDM}$ model [5]. Although, the $\Lambda \mathrm{CDM}$ is very successful in describing the large scale structure from CMB and other probes [6], a number of tensions have crept up in recent years, such as the Hubble constant tension between low redshift and high redshift probes [79], $\sigma_{8}$ tension [10], Lithium-7 problem in Big-Bang Nucleosynthesis [11], core-cusp and missing satellites problem $[12,13]$, failure to detect cold dark matter in the laboratory [14], failure to explain regularities in cosmic structure at galactic scales such as the radial acceleration relation and Baryonic Tully-Fisher relation [15, 16]. Therefore, a large number of works have tried to test the CDDR in a model-independent way using a variety of astrophysical probes [4, 17-31]. Any violation of CDDR would result in a value for $\eta(z)$ in Eq. 1, different from one. In such cases, $D_{L}$ can be expressed in terms of $D_{A}$ and $\eta(z)$, which is sometimes known as the deformed CDDR relation [32].

In this work, we shall use galaxy cluster observations observed in the microwave, via Sunyaev-Zeldovich effect (SZ, hereafter) and X-rays to test CDDR. Galaxy clusters are the most massive virialized collapsed objects in the

\footnotetext{
*E-mail: ph18resch11003@iith.ac.in

${ }^{\dagger}$ E-mail: shntn05@gmail.com
}

universe and are wonderful laboratories for a whole range of topics in Cosmology, galaxy evolution, and fundamental Physics [33-36]. The first test of CDDR using galaxy clusters was done by [17]. They used the X-ray and SZ observations from [37] to test the validity of the CDDR, and obtained a value for $\eta$ consistent with one at $2 \sigma$. Later, de Bernardis et al. [18] did a similar test using the angular diameter distances to 38 galaxy clusters from Xray observations with Chandra and SZ observations with the OVRO and BIMA interferometric arrays. Their results were consistent with no violation of CDDR at $1 \sigma$. The first model-independent cosmological test of CDDR was carried out in [38], by using joint SZ and X-ray surface brightness measurements, along with Type Ia supernovae (SNe) from the Constitution data [39]. Here, they used two different sample of galaxy clusters from [40] and [41], corresponding to elliptical and spherical geometry, respectively. For their analyses, two different parametric forms for $\eta(z)$ were tested. Their results for clusters with elliptical geometry were consistent with CDDR to within $2 \sigma$. For the sample with spherical geometry, discrepancy with CDDR at about $3 \sigma$ was reported. Hence, they concluded that the elliptical geometry of galaxy clusters is compatible with the validity of CDDR but not the spherical geometry. Gonçalves et al. [42] used X-ray $f_{\text {gas }}$ data, along with $\mathrm{SNe}$ Ia from the Union 2 compilation [43]. Here also, the same two samples of galaxy clusters [40, 41] were used. This work found that one of the samples [40] is consistent with CDDR, whereas the other sample [41] shows a $3.5 \sigma$ deviation from CDDR. Later, Holanda et al. [44] reported no violation of CDDR using a combination of SZ and X-ray gas mass fraction data [40]. Liang et al. [45] tested CDDR using a sample of 38 angular diameter distances from galaxy clusters assuming the spherical model [46], along with SNe Ia data from the Union 2 compilation [43]. They found no evidence for the violation of CDDR at $1-2 \sigma$, depending on the parameterization used. Yang et al. [47] carried out a model-independent test of CDDR using the angular diameter distances from two different cluster samples [46, 48] corresponding to spherical and elliptical geometry, respectively, in conjunction with the Union 2 SNe Ia sample [43]. How- 
ever, instead of using the SNe Ia $D_{L}$ from the distance modulus, they used the original $\mathrm{SNe}$ data for the restframe peak magnitudes and other parameters describing the influence of intrinsic color and reddening by dust. They obtained no violation of CDDR at $1 \sigma$ for both the elliptical and spherical cluster samples, but concluded that the spherical model [46] can better describe the intrinsic geometry of clusters as compared to the elliptical one [48], if the CDDR relation is valid. Santos-da-Costa et al. [49] used two different methods to test CDDR using 38 angular diameter distances obtained from [46] (which was also used in earlier tests of CDDR [47]), along with $f_{\text {gas }}$ measurements [40] spanning the redshift range $0.14<z<0.89$. This was then compared with an independent estimate of the angular diameter distance, reconstructed using $H(z)$ data from cosmic chronometers and BAO. In this work, the departure from CDDR was reconstructed in a non-parametric manner. Both the methods were consistent with the validity of CDDR at $1 \sigma$. Holanda and Pereira [50] studied the temporal variation of the fine structure constant, modified distance duality relation, and the modified evolution law of the cosmic microwave background radiation by using the angular diameter distances to 29 galaxy clusters [46], Union 2.1 Type Ia SNe compilation [51], and $38 T_{C M B}$ measurements $[52,53]$. All of these variants/modifications of the Standard Model arise naturally from a class of modified gravity theories, which break the Einstein Equivalence Principle. Their results are consistent with no violation of these laws at $1 \sigma$. (See also $[54,55]$ for similar followup studies). Holanda et al. [32] (H19, hereafter) have also done a study of the validity of CDDR relation using the $Y_{S Z}-Y_{X}$ scaling relation for 61 Planck ESZ clusters jointly with deep XMM-Newton archive observations in the redshift range $0.044 \leqslant z \leqslant 0.444$ [56], along with Type Ia SNe from the Pantheon compilation [57]. They reported no violation of CDDR at $1.5 \sigma$. Most recently, da Silva et al. [58] did a Bayesian model comparison assuming CDDR violation for different forms of $\eta(z)$ using two sets of data namely SNe Ia [57] + angular diameter distances from galaxy clusters [48] and SNe Ia [57] + gas mass fraction data [59]. Their results showed agreement with CDDR at $2 \sigma$ for SNe Ia and the angular diameter distances from galaxy clusters sample, and at $1 \sigma$ for $\mathrm{SNe}$ Ia along with the gas mass fraction measurements.

In this work, we shall use SZ selected clusters from the South Pole telescope in conjunction with X-ray followup observations from the XMM-Newton telescope to test for a violation of CDDR. This paper is structured as follows. In Sec. II , we briefly explain the basic theory behind the SZ and X-ray observables used to test CDDR. In Sec. III, we describe our cluster sample. Sec. IV discusses the calculation of $D_{L}$ using three independent methods.
Details of our analysis and results can be found in Sec. V. We conclude in Sec. VI.

\section{METHODOLOGY}

The Inverse Compton scattering between the CMB photons and the hot electrons present in the intra-cluster medium causes a spectral distortion of the CMB black body spectrum [60-63]. This spectral distortion is known as the SZ effect. It is nearly independent of redshift, and hence can detect galaxy clusters upto very high redshifts. The distortion is characterized by a parameter, called Compton- $y$ parameter, which is given by [61, 62]:

$$
y=\frac{\sigma_{T} k_{B}}{m_{e} c^{2}} \int n_{e} T d l
$$

where, $T$ is the electron temperature, $m_{e}$ is mass of the electron, $c$ is the speed of light, $n_{e}$ is the electron number density, $k_{B}$ is the Boltzmann constant, and $\sigma_{T}$ is the Thompson scattering cross section which is given by,

$$
\sigma_{T}=\frac{8 \pi}{3}\left(\frac{\epsilon^{2}}{m_{e} c^{2}}\right)^{2}=\frac{8 \pi}{3}\left(\frac{\hbar^{2} \alpha^{2}}{m_{e}^{2} c^{2}}\right)
$$

If we model the variation in $\alpha$ as $\alpha(z) \equiv \alpha_{0} \phi(z)$, where $\alpha_{0}$ is the present value of $\alpha$, the fractional variation in $\alpha$ can be written as,

$$
\frac{\Delta \alpha}{\alpha_{0}}=\phi(z)-1
$$

We assume that the intra-cluster medium is an ideal gas, with its equation of state given by $P=n_{e} k_{B} T$, where $P$ refers the pressure of the intra-cluster gas. The relation between the integrated Compton parameter over the solid angle $\left(Y_{S Z}\right)$ and the angular diameter distance to the cluster $\left(D_{A}\right)$ is given by [64]:

$$
Y_{S Z} D_{A}^{2} \propto \phi(z)^{2}
$$

The hot intra-cluster gas emits X-rays mainly through thermal bremsstrahlung emission [33, 65]. If $M_{g}(R)$ is the gas mass within radius $R$ and $T_{X}$ is the X-ray temperature, then the average thermal energy of the cluster gas is given by [66],

$$
Y_{X}=M_{g}(R) T_{X}
$$

An exact expression for the gas mass $M_{g}(<R)$ within a radius $R$ can be obtained by assuming that the number density obeys the spherical- $\beta$ model [65] and X-ray emission via thermal bremsstrahlung, and is given by [67] 


$$
M_{g}(<R)=m_{H}\left(\frac{3 \pi \hbar m_{e} c^{2}}{2(1+X) e^{6}}\right)^{1 / 2}\left(\frac{3 m_{e} c^{2}}{2 \pi k_{B} T_{e}}\right)^{1 / 4} \frac{1}{\left[\overline{g_{B}}\left(T_{e}\right)\right]^{1 / 2}} r_{c}^{3 / 2}\left[\frac{I_{M}\left(R / r_{c}, \beta\right)}{I_{L}^{1 / 2}\left(R / r_{c}, \beta\right)}\right]\left[L_{X}(<R)\right]^{1 / 2}
$$

We then absorb the terms involving $e, c$, and $\hbar$ into $\alpha$, and rewrite this expression in terms of $\alpha \equiv \alpha_{0} \phi(z)$ (from
Eq. 4) as follows:

$$
M_{g}(<R)=\alpha_{0} \phi(z)^{-3 / 2} m_{H}\left(\frac{3 \pi m_{e}}{2(1+X) \hbar^{2}}\right)^{1 / 2}\left(\frac{3 m_{e}}{2 \pi k_{B} T_{e}}\right)^{1 / 4} \frac{1}{\left[\overline{g_{B}}\left(T_{e}\right)\right]^{1 / 2}} r_{c}^{3 / 2}\left[\frac{I_{M}\left(R / r_{c}, \beta\right)}{I_{L}^{1 / 2}\left(R / r_{c}, \beta\right)}\right]\left[L_{X}(<R)\right]^{1 / 2},
$$

In Eq. 8, $L_{X}(<R)$ is the total X-ray luminosity; $X$ is the mass fraction of hydrogen, $r_{c}$ indicates the core radius; $T_{e}$ is the gas temperature, $\overline{g_{B}}\left(T_{e}\right)$ the Gaunt factor; and $I_{M}, I_{L}$ are defined as follows [67]:

$$
\begin{gathered}
I_{M}\left(R / r_{c}, \beta\right) \equiv \int_{0}^{R / r_{c}}\left(1+x^{2}\right)^{-3 \beta / 2} x^{2} d x \\
I_{L}\left(R / r_{c}, \beta\right) \equiv \int_{0}^{R / r_{c}}\left(1+x^{2}\right)^{-3 \beta} x^{2} d x .
\end{gathered}
$$

$L_{X}, r_{c}$, and $R$ are not direct observables, but depend on the underlying cosmological model and are given as [67]:

$$
\begin{aligned}
L_{X}(<R) & =4 \pi D_{L}^{2} f_{X}(<\theta), \\
r_{c} & =\theta_{c} D_{A}, \\
R & =\theta D_{A},
\end{aligned}
$$

where $f_{X}(<\theta)$ is the total bolometric flux within the outer angular radius $\theta$, and $\theta_{c}$ is the core angular radius. Therefore, from Eq. 8, Eq. 9, and Eq. 10 we find that $M_{g}(R)$ scales with $\phi(z), D_{L}$, and $D_{A}$ according to:

$$
M_{g}(<\theta) \propto \phi(z)^{-3 / 2} D_{L} D_{A}^{3 / 2},
$$

$D_{L}$ and $D_{A}$ are connected via CDDR (cf. Eq. 1). If we parameterize a violation of CDDR using the deformed CDDR $D_{L} \equiv \eta(z)(1+z)^{2} D_{A}$, then $M_{g}$ and consequently, $Y_{X}$ scales with $\alpha$ and $\eta(z)$ as $[64,68]$,

$$
Y_{X} \propto M_{g} \propto \phi(z)^{-3 / 2} \eta(z)
$$

For a wide class of modified theories of gravity [69-71], Einstein's equivalence principle breaks down due to the coupling of the scalar field with the electromagnetic sector. In these theories, $\alpha(z)$ (and thereby $\phi(z))$ and $\eta(z)$ are intertwined and related according to $\phi(z)=(\eta(z))^{2}$. $Y_{S Z}$ and $Y_{X}$ can then be rewritten in terms of $\eta(z)$ as:

$$
Y_{S Z} D_{A}^{2} \propto \eta(z)^{4}
$$

and

$$
Y_{X} \propto \eta(z)^{-2}
$$

$Y_{S Z}$ and $Y_{X}$ are two different proxies for the thermal energy of the cluster [72], so the ratio $Y_{S Z} D_{A}^{2} / C_{X S Z} Y_{X}$ is expected be constant with redshift, since both these quantities scale with redshift and mass in exactly the same way. Simulations show that this ratio is constant with $5-15 \%$ scatter [73-77]. The ratio would be exactly one for clusters with isothermal or universal temperature profiles [68, 78, 79]. From Eq. 14 and 15, for any violations of CDDR, this ratio scales with $\eta(z)$ as:

$$
\frac{Y_{S Z} D_{A}^{2}}{Y_{X} C_{X S Z}}=C \eta(z)^{6}
$$

where $C_{X S Z} \approx 1.416 \times 10^{-19}\left(\frac{M p c^{2}}{M_{\odot} k e V}\right)$ and $C$ is an arbitrary constant, which contains all the cluster astrophysics and is equal to one for an isothermal profile [68].

From Eq. 6 and 12, one can see that $Y_{X}$ scales with $D_{A}$ according $Y_{X} \propto D_{A}^{5 / 2}$, if we assume a flat $\Lambda \mathrm{CDM}$ cosmology and the validity of CDDR. Following H19, we multiply $Y_{X}$ by $D_{A}^{5 / 2} /\left(D_{A}^{r e f}\right)^{5 / 2}$, where $D_{A}^{r e f}$ is the angular diameter distance in the fiducial reference $\Lambda \mathrm{CDM}$ model, corresponding to $\Omega_{M}=0.3$ and $\Omega_{\Lambda}=0.7$. This eliminates the dependence of $M_{g}(R)$, with respect to the fiducial cosmology. Hence we get,

$$
\frac{Y_{S Z} D_{A}^{2}\left(D_{A}^{r e f}\right)^{5 / 2}}{Y_{X} C_{X S Z} D_{A}^{5 / 2}}=C \eta(z)^{6}
$$

Since we want to carry out a test of CDDR in a modelagnostic fashion, we recast this equation in terms of $D_{L}$ by parameterizing any violation of CDDR using $\eta(z)$ defined in Eq. 1. So Eq. 17 can be written as,

$$
\frac{Y_{S Z}\left(D_{A}^{r e f}\right)^{5 / 2}(1+z)}{Y_{X} C_{X S Z} D_{L}^{1 / 2}}=C \eta(z)^{11 / 2}
$$




\section{CLUSTER SAMPLE}

For our analysis, we use $Y_{S Z}$ and $Y_{X}$ for 58 SPT-SZ selected galaxy clusters from Bulbul et al. [80]. The SZ data have been taken from the South Pole Telescope (hereafter SPT) which is a $10 \mathrm{~m}$ millimeter wave telescope located at the South Pole [81]. One of the main goals of the SPT is to find galaxy clusters using their SZ signatures upto very high redshifts. SPT has imaged the sky at three different frequencies viz. $95 \mathrm{GHz}, 150$ $\mathrm{GHz}$, and $220 \mathrm{GHz}$ [81], and carried out a 2500 square degree survey between 2007 to 2011. A total of 516 galaxy clusters have been detected in this survey, with a mass threshold of $3 \times 10^{14} M_{\odot}$ upto a redshift of $1.8[82,83]$. Many dedicated optical surveys, e.g. DES [84], BCS [85] as well as targeted observations [86] have followed-up the SPT cluster candidates in order to determine confirm these as true clusters and determine their redshifts. Here, we have also used the X-ray observations (by XMMNewton) for 58 of these clusters, in the redshift range $0.2 \leq z \leq 1.5$ [80]. The $Y_{X}$ parameter from XMMNewton measurements has been measured at $R_{500}$. The $Y_{S Z}$ measurements provided by SPT have been obtained by averaging over a cylindrical volume. These need to be transformed to a spherical volume with radius equal to $R_{500}$, since $Y_{X}$ measurements are provided at $R_{500}$. The details of the conversion procedure are discussed in the Appendix.

\section{CALCULATION OF LUMINOSITY DISTANCE $D_{L}$}

We use three different methods to estimate the the luminosity distance, $D_{L}$ for each cluster. We now describe each of these methods.

\section{A. Method 1: Using a redshift cut}

We use Type Ia SNe sample from the Union 2.1 compilation [51], which consists of 580 Type Ia supernovae data spanning the redshift range $0.015 \leqslant z \leqslant 1.414$ (with median redshift of $z \approx 0.294$ ) in order to estimate $D_{L}$ for the SPT-SZ sample by choosing the closest SNe corresponding to every cluster. Similar to H19, we use the following cut on the supernova redshift $\left(z_{S N e}\right)$ for a cluster with redshift $z:\left|z-z_{S N e}\right| \leq 0.005$. For most galaxy clusters, there are multiple supernovae satisfying the above cut. To determine the $D_{L}$ and its error at the cluster location in such cases, we first need to evaluate the weighted average of the distance modulus of Type Ia SNe $(\bar{\mu}(z))$ and its associated error.

To reconstruct $D_{L}$ from the distance modulus $\mu$, we start from the following equation [87]:

$$
\mu=m-M=5 \log _{10}\left(\frac{d_{L}}{10(p c)}\right)
$$

The Type Ia SNe surveys cannot directly measure $\mu$, as they only measure the relative distances. The distance modulus for the Union 2.1 catalog $^{1}$, has obtained by assuming $M=-19.31$ and $h=0.7$, where $h \equiv H_{0} / 100$. Therefore, we can re-write $\mu$ as a function of $h$ as follows:

$$
\mu=m-M=m-\left[-19.31+5 \log _{10}\left(\frac{h}{0.7}\right)\right]
$$

Currently, there are discrepancies between the different measurements of $H_{0}$, with a fierce ongoing debate on whether this is a harbinger of new Physics or only systematics [7]. The tension between the model-independent low redshift based probes and those estimated using early time probes with the $\Lambda \mathrm{CDM}$ model ranges between 4-6 $\sigma$. (See [7-9] for extensive reviews of these different measurements). Since the $\mu$ obtained from Type Ia SNe measurements has an explicit dependence on $h$, we consider it as a nuisance parameter and marginalize over $h$, while testing for deviations from CDDR. This is similar to the analysis done in [88]. For this purpose, we use a Gaussian prior on $h$, using the latest $H_{0}$ measurement from the SH0ES team of $H_{0}=73.2 \pm 1.3 \mathrm{~km} / \mathrm{sec} / \mathrm{Mpc}$ [89]. The reason is that the SHOES measurement is agnostic to details of the underlying $\Lambda \mathrm{CDM}$ model, and is based on directly measuring the distance-redshift relation by constructing a distance ladder.

One then invert Eq. 19 to obtain $D_{L}$ as a function of $h$ using the $\mu$ provided for the Union 2.1 catalog

$$
D_{L}(z)(\mathrm{Mpc})=\left(\frac{0.7}{h}\right) 10^{(\mu-25) / 5}
$$

In case of multiple matches for a given redshift, we use the weighted average $\bar{\mu}(z)$ in the above equation. We then plug Eq. 21 in Eq. 18.

For six clusters, we could not find any supernovae counterparts with the aforementioned redshift cut, and these clusters were therefore culled from our sample. Thereafter, we are left with a sample of 52 clusters for our analysis.

\section{B. Method 2: Non-parametric reconstruction using Type Ia SNe}

We now adopt another method to find $D_{L}$ for every cluster, by using the Gaussian Processes Regression (GPR). A Gaussian process is the generalization of a Gaussian distribution. It is characterized by a mean and a covariance function (usually called the kernel function) [90]. More details about GPR can be found in our previous works [91, 92], and references therein. For this

\footnotetext{
1 This catalog has been downloaded from http://supernova.lbl. gov/Union/figures/SCPUnion2.1_mu_vs_z.txt
} 
work, we select the squared exponential (RBF) covariance function, which is given by:

$$
K(x, \tilde{x})=\sigma_{f}^{2} \exp \left[\frac{-(x-\tilde{x})^{2}}{2 l^{2}}\right],
$$

It depends on two hyperparameters: $\sigma_{f}$ and $l$. The length parameter $l$ controls the smoothness of the kernel function. To reconstruct $D_{L}$ at the cluster redshift, we used the scikit-learn module in python [93]. Fig 1 shows the reconstructed luminosity distance as a function of $z$ using GPR. Using this non-parametric reconstruction, one can estimate $D_{L}$ for every SPT-SZ cluster. In order to be consistent with Method 1, we omit the same six clusters, for which $D_{L}$ could not be estimated using Method 1. Similar to Method 1, $D_{L}$ reconstructed using GPR from the Union 2.1 sample also needs to be rescaled as a function of $h$, while testing for CDDR violation, in order to marginalize over $h$.

\section{Method 3: Reconstruction using cosmic chronometers}

Here, we used $31 H(z)$ measurements from Li et al. [94] (same as that used in [91, 92]) spanning the redshift range $0.07 \leqslant z \leqslant 1.965$. These $H(z)$ measurements are obtained from cosmic chronometers, which provide a model agnostic estimate of the expansion history at any redshift $z$ [95]. Using these $H(z)$ measurements, we have again used the scikit-learn module for GPR, to estimate $H(z)$ for any redshift. Furthermore, the reconstructed $H(z)$ data can be used to derive the luminosity distance $D_{L}$ which can be written as,

$$
D_{L}(z)=c(1+z) \int_{0}^{z} \frac{d z^{\prime}}{H\left(z^{\prime}\right)}
$$

where $H\left(z^{\prime}\right)$ is the reconstructed Hubble data. Fig 2 shows the reconstructed $H(z)$ data from chronometers as a function of $z$ along with their $1 \sigma$ and $2 \sigma$ uncertainties. Note that, here too, the same six clusters as in Method 1 have been omitted.

A comparison plot of the luminosity distance $D_{L}$ as a function of $z$ using all the three methods for the 52 SPT-SZ clusters is shown in Fig 3. The fractional median difference between the luminosity distance $D_{L}$ obtained by Method 1, 2, and 3, when compared against each other is equal to $2.3 \%, 5.4 \%, 2.9 \%$ between Method 1-2, Method 2-3, and Method 1-3 respectively. Therefore, this difference in $D_{L}$ between the three methods is marginal.

\section{ANALYSIS AND RESULTS}

In order to constrain $\eta(z)$ (as used in Eq. 18), we use the following parametric forms [69]. These were also used in H19 and other previous works testing for a violation of CDDR.

$$
\begin{aligned}
& \eta(z)=1+\eta_{0} z \\
& \eta(z)=1+\eta_{0}[z /(1+z)] \\
& \eta(z)=1+\eta_{0} \ln (1+z) \\
& \eta(z)=(1+z)^{\eta_{0}}
\end{aligned}
$$

For all the above parametrizations, $\eta_{0}$ encapsulates a possible departure from the standard CDDR, and $\eta_{0}=0$ corresponds to no CDDR violation. Table I summarizes all possible combinations of the different methods used for $D_{L}$ measurements and the multiple $\eta(z)$ parametrizations, we have analyzed in this work. The $Y_{S Z}-Y_{X}$ ratio given by Eq.18 is shown in Fig 4 for each of the methods used for reconstructing $D_{L}$ (as discussed in Sec. IV).

We now proceed to obtaining constraints on $\eta(z)$ for each of the parametrizations. The likelihood function $\mathcal{L}$ used in our analysis is given by:

$$
-2 \ln \mathcal{L}=\sum_{i=1}^{N} \frac{\left(\eta_{\text {obs }}\left(z_{i}\right)-C \eta(z)^{11 / 2}\right)^{2}}{\sigma_{i}^{2}}+\sum_{i=1}^{N} \ln 2 \pi \sigma_{i}^{2},
$$

where $\eta_{\text {obs }}$ is obtained from Eq. 18 and $\sigma_{i}$ denotes the total error calculated as follows:

$$
\sigma_{i}^{2}=\sigma_{\eta}^{2}+\sigma_{i n t}^{2}
$$

where, $\sigma_{\eta}^{2}$ represents the error in $\eta_{\text {obs }}$ which is calculated by propagating the errors in $Y_{S Z}, Y_{X}, D_{L}(z)$, and $D_{A}^{r e f}$. When we estimate $D_{L}$ using Method 1 and Method 2, it will also have an additional dependence on $h$ (cf. Eq. 21). Therefore, any estimate on the violation of CDDR using the $D_{L}$ obtained from these two methods will contain an additional free parameter, i.e., $h$ which has to be marginalized over in order to constrain the CDDR parameters. As mentioned earlier, we use a Gaussian prior on $h$, with a mean equal to 0.732 and scale parameter of 0.0013 We have also included an intrinsic scatter term $\left(\sigma_{i n t}\right)$ as a free parameter, which is added in quadrature to the observational error, while maximizing the likelihood. We used the emcee MCMC sampler [96] to estimate the model parameters i.e. $\eta_{0}$ and $C$, by maximizing the likelihood function defined in Eq. 28.

The marginalized one-dimensional posteriors for each of the parameters along with the 2-D 68\%, 95\%, and $99 \%$ credible intervals, are displayed in Fig. 5- 7 for Eq. 24, corresponding to Methods 1, 2, and 3, respectively for calculating $D_{L}$. Note that in Fig 5 and Fig. 6, we need to marginalize over $h$, since $D_{L}$ has been estimated using Method 1 and Method 2. However, there is no dependence on $h$ in Method 3, and hence Fig. 7 does not contain any contours for $h$. A complete summary of all our results for each of the four parametric forms using all the three methods for computing $D_{L}$ is tabulated in Table II. This table also contains a summary of previous 


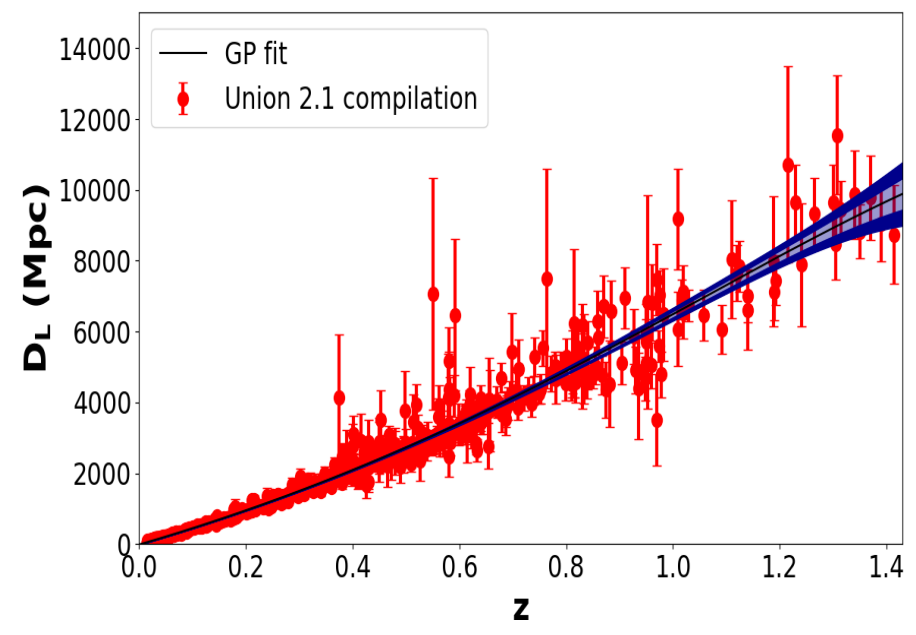

FIG. 1: Reconstruction of $D_{L}$ using GPR. The red data points show the 580 Type Ia SNe taken from Union 2.1 compilation [51]. The black line indicates the best GP fit to the data along with $1 \sigma$ and $2 \sigma$ error bands shown by the two different shades of blue color. For this plot we have assumed $h=0.7$.

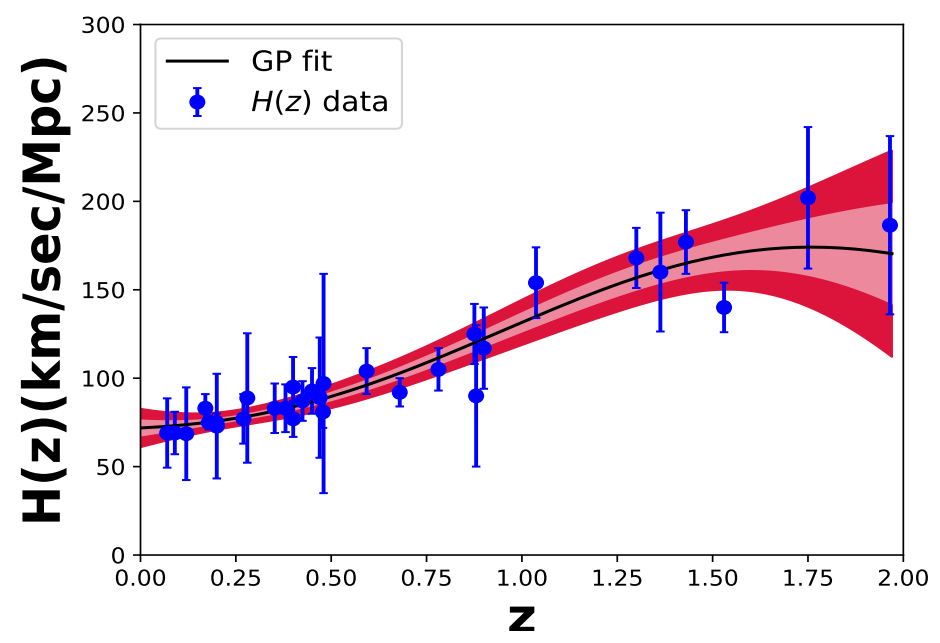

FIG. 2: Reconstruction of $H(z)$ using GPR. The blue data points represent the $31 H(z)$ cosmic chronometer measurements compiled by Li et al. [94]. The black line indicates the best GP fit to data along with $1 \sigma$ and $2 \sigma$ error bands shown by the two different shades of red color.

results in literature for the same parametric forms, whenever available. A graphical summary of all our results for the different cases can be found in Fig 8. Therefore, the results from all these analyses indicate that there is no violation of CDDR, and the parameter which encapsulates any violation of CDDR as a function of redshift is consistent with zero to within $1 \sigma$ for all the parametric forms used.

\section{CONCLUSIONS}

In this work, we carried out a model-independent test of CDDR, along the same lines as H19. For this purpose, we used 52 SPT-SZ clusters in the redshift range $0.2 \leq z \leq 1.5$ in conjunction with X-ray measurements from XMM-Newton [80]. To get independent distance measurements at the cluster redshifts, we used $D_{L}$ from Union 2.1 Type Ia SNe sample [51] and $H(z)$ measurements from cosmic chronometers [94]. The cluster observables used to test CDDR include the SZ Comptoionization parameter $\left(Y_{S Z}\right)$, along with its X-ray counterpart $\left(Y_{X}\right)$, both of which are independent thermal energy 


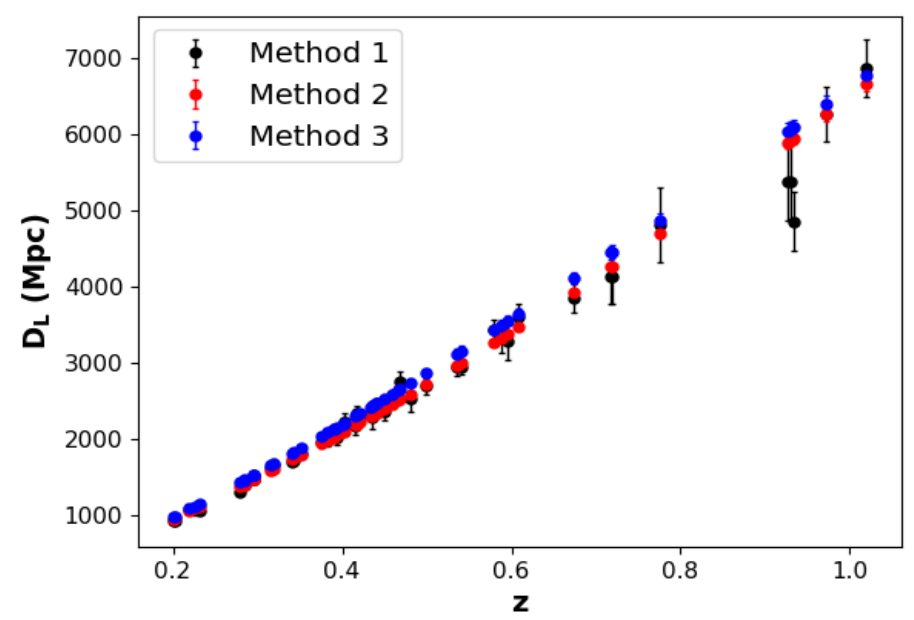

FIG. 3: A comparison of $D_{L}$ for the SPT cluster sample obtained from the different methods as discussed in Sec IV. The fractional median difference between the luminosity distance $D_{L}$ obtained by Method 1, 2, and 3, when compared against each other is equal to $2.3 \%, 5.4 \%, 2.9 \%$ respectively and hence is negligible. Note that the $D_{L}$ values for Method 1 and 2 in this plot are obtained by assuming $h=0.7$.

\begin{tabular}{|l|c|c|c|c|c|c|c|}
\hline Combination & Method 1 & Method 2 & Method 3 & Eq. 24 & Eq. 25 & Eq. 26 & Eq. 27 \\
\hline Case I & $\checkmark$ & & & $\checkmark$ & & & \\
Case II & $\checkmark$ & & & & $\checkmark$ & & \\
Case III & $\checkmark$ & & & & & $\checkmark$ & $\checkmark$ \\
Case IV & $\checkmark$ & & & & & & \\
Case V & & $\checkmark$ & & $\checkmark$ & & & \\
Case VI & & $\checkmark$ & & & $\checkmark$ & $\checkmark$ & \\
Case VII & & $\checkmark$ & & & & $\checkmark$ \\
Case VIII & & $\checkmark$ & & & & & $\checkmark$ \\
Case IX & & & $\checkmark$ & $\checkmark$ & & & \\
Case X & & & $\checkmark$ & & $\checkmark$ & $\checkmark$ & \\
Case XI & & & $\checkmark$ & & & $\checkmark$ & $\checkmark$ \\
Case XII & & & $\checkmark$ & & & \\
\hline
\end{tabular}

TABLE I: An illustration of the different analyses used for testing CDDR.

\begin{tabular}{|c|c|c|c|c|c|}
\hline Dataset used & Eq. 24 & Eq. 25 & Eq. 26 & Eq. 27 & Reference \\
\hline Angular Dia Dist+SNe Ia & $-0.28 \pm 0.44(2 \sigma)$ & $-0.43 \pm 0.60(2 \sigma)$ & - & - & {$[38]$} \\
\hline Angular Dia Dist+SNe Ia & $-0.15 \pm 0.17$ & $-0.23 \pm 0.24$ & - & - & [97] \\
\hline Angular Dia Dist+SNe Ia & $-0.07 \pm 0.19$ & $-0.11 \pm 0.26$ & - & - & [20] \\
\hline$f_{\text {gas }}$ & $-0.06 \pm 0.16$ & $-0.07 \pm 0.24$ & - & - & {$[44]$} \\
\hline$f_{\text {gas }}+\mathrm{SNe} \mathrm{Ia}$ & $-0.03_{-0.65}^{+1.03}$ & $-0.08_{-1.22}^{+2.28}$ & - & - & {$[42]$} \\
\hline Angular Dia Dist+SNe Ia & $-0.23 \pm 0.23(2 \sigma)$ & $-0.35 \pm 0.37(2 \sigma)$ & - & - & {$[45]$} \\
\hline Angular Dia Dist+SNe Ia & $0.16_{-0.39}^{+0.56}$ & - & - & - & {$[47]$} \\
\hline Angular Dia Dist $+H(z)$ & $-0.10_{-0.13}^{+0.12}$ & $-0.16_{-0.19}^{+0.18}$ & - & - & [49] \\
\hline$f_{g a s}+H(z)$ & $0.062_{-0.15}^{+0.17}$ & $-0.17_{-0.28}^{+0.34}$ & - & - & [49] \\
\hline Angular Dia Dist $+f_{\text {gas }}+\mathrm{SNe} \mathrm{Ia}+T_{C M B}$ & $-0.012 \pm 0.022$ & $-0.02 \pm 0.034$ & $-0.017 \pm 0.027$ & $-0.017 \pm 0.026$ & [55] \\
\hline Angular Dia Dist $+f_{\text {gas }}+\mathrm{SNe} \mathrm{Ia}+T_{C M B}$ & $-0.011 \pm 0.021$ & $-0.015 \pm 0.033$ & $-0.013 \pm 0.027$ & $-0.013 \pm 0.028$ & [55] \\
\hline Strong Grav. Lensing+SNe Ia+GRBs & $0.00 \pm 0.10(2 \sigma)$ & $-0.36_{-0.42}^{+0.37}(2 \sigma)$ & $-0.10 \pm 0.24(2 \sigma)$ & $-0.16_{-0.51}^{+0.24}(2 \sigma)$ & {$[54]$} \\
\hline Strong Grav. Lensing+SNe Ia+GRBs & $0.15 \pm 0.13(2 \sigma)$ & $-0.18_{-0.65}^{+0.45}(2 \sigma)$ & $0.22_{-0.32}^{+0.40}(2 \sigma)$ & $0.27_{-0.38}^{+0.22}(2 \sigma)$ & {$[54]$} \\
\hline$Y_{S Z}-Y_{X}+\mathrm{SNe} \mathrm{Ia}$ & $0.05 \pm 0.07(2 \sigma)$ & $0.09 \pm 0.16(2 \sigma)$ & - & - & {$[32]$} \\
\hline$Y_{S Z}-Y_{X}$ ratio+SNe Ia(Method 1$)$ & $0.009 \pm 0.05$ & $0.021 \pm 0.11$ & $0.012 \pm 0.07$ & $0.009 \pm 0.07$ & This work \\
\hline$Y_{\mathrm{SZ}}-\mathrm{Y}_{\mathrm{X}}$ ratio+SNe Ia(Method 2$)$ & $0.008 \pm 0.05$ & $0.017 \pm 0.11$ & $0.015 \pm 0.07$ & $0.010 \pm 0.07$ & This work \\
\hline$Y_{\mathrm{SZ}}-\mathrm{Y}_{\mathrm{X}}$ ratio $+H(z)($ Method 3$)$ & $0.008 \pm 0.05$ & $0.019 \pm 0.11$ & $0.013 \pm 0.07$ & $0.010 \pm 0.07$ & This work \\
\hline
\end{tabular}

TABLE II: Constraints on $\eta_{0}$ from previous studies using different parametric forms as defined in Eq. 24 - Eq. 27 along with our results presented in last three rows. The quoted uncertainties are at $1 \sigma$ wherever not mentioned explicitly. We do not find any violation of CDDR from our analyses. 


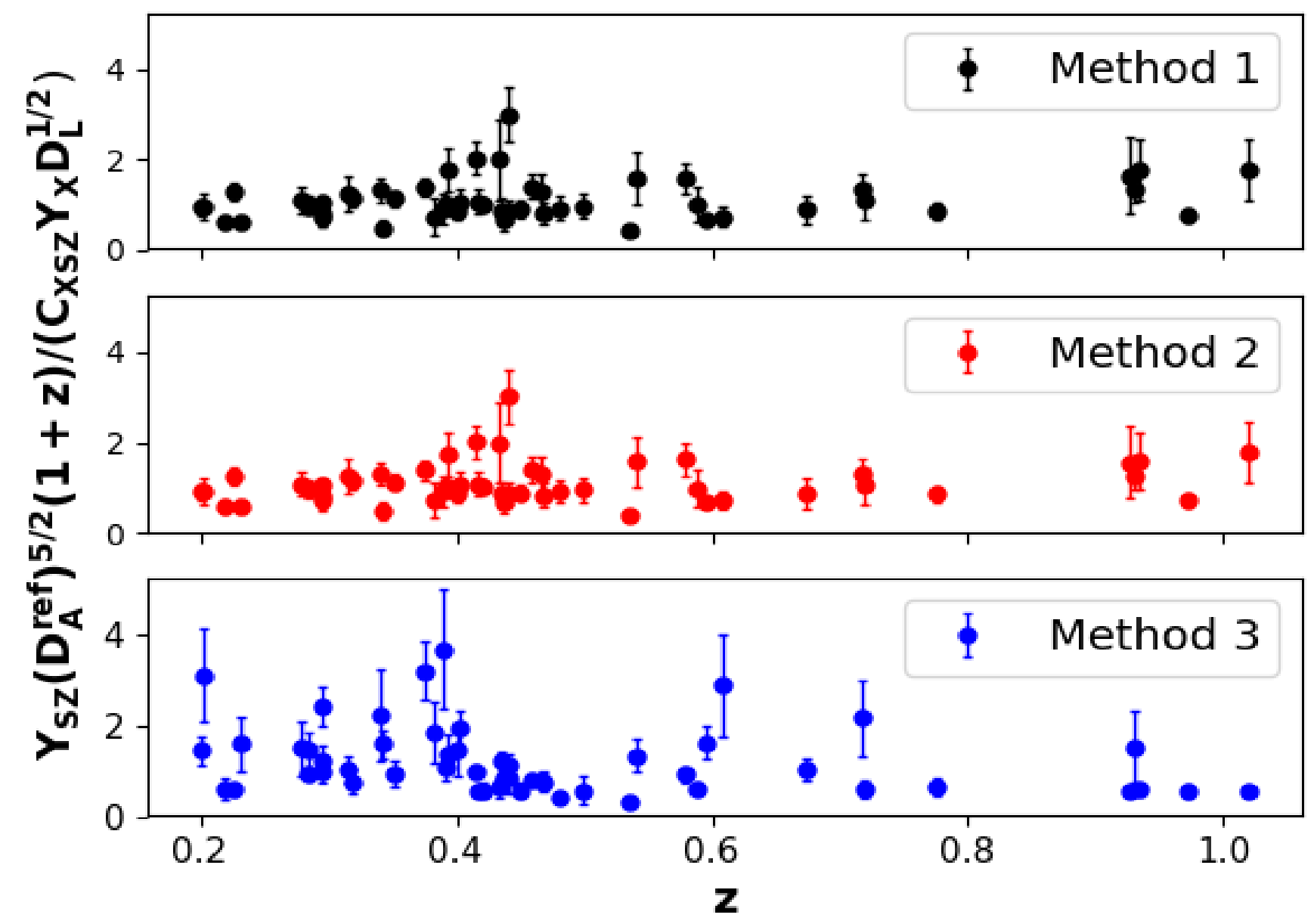

FIG. 4: A comparison of the $Y_{S Z^{-}} Y_{X}$ ratio defined in Eq. 18 for the three different methods used for calculating $D_{L}$, as described in Sec. IV. Note that for making this plot for Method 1 and Method 2, we have used $h=0.7$. 


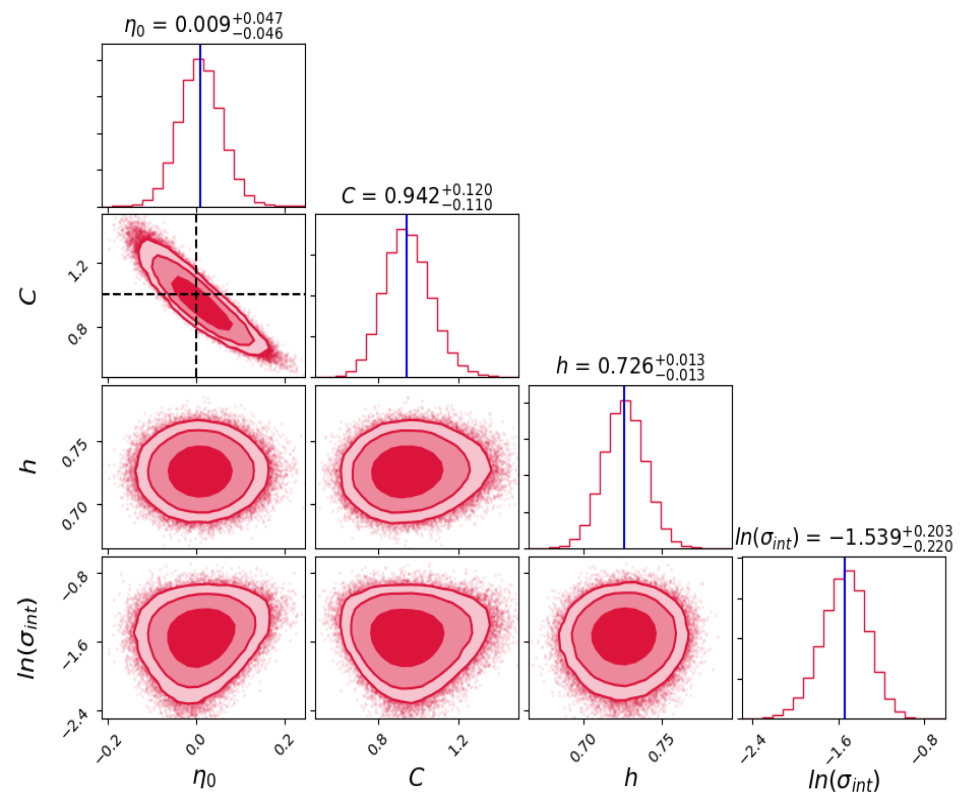

FIG. 5: Case I: Constraints on the parameters $\eta_{0}, C$, and $h$ along with $\ln \left(\sigma_{\text {int }}\right)$. The plots along the diagonal are the onedimensional marginalized likelihood distributions. The contour plot represents the two-dimensional marginalized constraints showing the $68 \%, 95 \%$, and $99 \%$ credible regions. These contours have been obtained using the Corner python module [98].

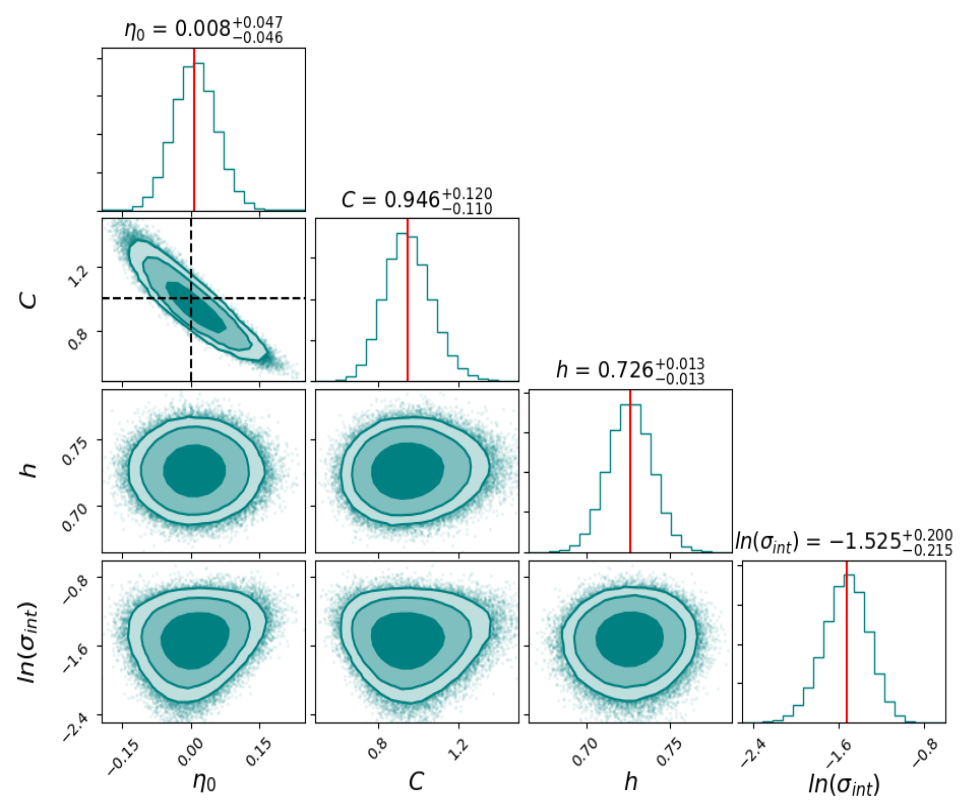

FIG. 6: Case V: Constraints on the parameters $\eta_{0}, C$, and $h$ along with $\ln \left(\sigma_{\text {int }}\right)$. The plots along the diagonal are the onedimensional marginalized likelihood distributions. The contour plot represents the two-dimensional marginalized constraints showing the $68 \%, 95 \%$, and $99 \%$ credible regions. These contours have been obtained using the Corner python module [98].

proxies, having different scalings with $\eta(z)$ (cf. Eq. 14 and 15). The $Y_{S Z}$ to $Y_{X}$ ratio is then used to test the CDDR, which is given by Eq. 18 . One input needed for this analysis is the luminosity distance at the cluster redshift, for which we have used three independent estimators. These are summarized in Sect IV and tabulated in Table I.

The dimensionless ratio of $Y_{S Z}$ to $Y_{X}$ (after suitable scalings) is shown in Fig 4 as a function of $z$ for all the three methods used for estimating $D_{L}$.

To test the validity of CDDR, we chose different parametric forms, defined in Eqs. 24 - Eq. 27, where the parameter $\eta_{0}$ characterizes any departure from CDDR. Table I shows all the possible used cases studied in this work. Furthermore, to estimate the parameters in Eqs. 24 - Eq. 27, we maximize the likelihood function 


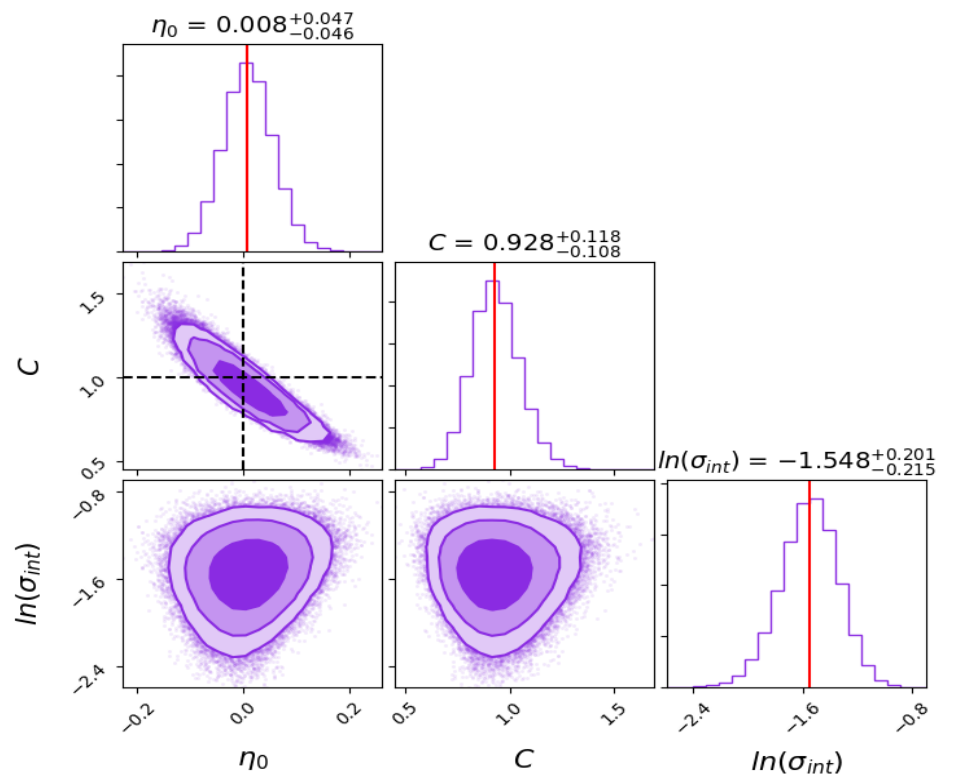

FIG. 7: Case IX: Constraints on the parameters $\eta_{0}$, and $C$ along with $\ln \left(\sigma_{\text {int }}\right)$. The plots along the diagonal are the onedimensional marginalized likelihood distributions. The contour plot represents the two-dimensional marginalized constraints showing the $68 \%, 95 \%$, and $99 \%$ credible regions. These contours have been obtained using the Corner python module [98]. Note that unlike Fig. 5 and Fig. 6, there is no contour for $h$, since $D_{L}$ estimated using this method (Method 3) does not depend on $h$.

given by Eq 28. Two of the three methods obtain the $D_{L}$ value from the Union 2.1 catalog, which assumed $h=0.7$. While using these $D_{L}$ values, we marginalized over $h$, using a Gaussian prior over $h$ from the latest SH0ES measurement [89], in order to obtain marginalized constraints on the CDDR violating parameters.

Our results for all these analyses can be found in Table II along with a comparison with previous results. A graphical summary of all our results for CDDR (for all the cases studied herein) can be found in Fig 8. For Cases I, V, and IX, which test the parametric form in Eq. 24 using Methods 1, 2, and 3, respectively the joint 2-D credible intervals for $\eta_{0}, C$ (a parameter in the $Y_{S Z}$ to $Y_{X}$ ratio) and $h$ (if needed) can be found in Fig 5 - Fig 7. Our results from all these analyses are consistent with
CDDR within $1 \sigma$. Therefore, we conclude that there is no evidence for a violation of CDDR.

\section{APPENDIX}

The $Y_{S Z}$ measurements provided by SPT have been obtained by averaging over a cylindrical volume within an aperture radius of $0.75 R^{\prime}$ [82]. Since, the $Y_{S Z}$ parameter defined in Eq. 14 needs to be averaged over a spherical volume, we need to convert the cylindrically averaged $Y_{S Z}$ (provided by SPT) to a spherically averaged $Y_{S Z}$ at $R_{500}$, for which we have used the following conversion method [99, 100]:

$$
\begin{aligned}
Y_{c y l}\left(R_{1}\right) & =Y_{s p h}\left(R_{b}\right)-\frac{\sigma_{T}}{m_{e} c^{2}} \int_{R_{1}}^{R_{b}} 4 \pi P(r) \sqrt{r^{2}-R_{1}^{2}} r d r \\
Y_{s p h}\left(R_{2}\right) & =\frac{\sigma_{T}}{m_{e} c^{2}} \int_{0}^{R_{2}} 4 \pi P(r) r^{2} d r
\end{aligned}
$$

where the subscripts $c y l$ and $s p h$ denote the $Y_{S Z}$ parameter, measured in cylindrical and spherical volumes, respectively. $R_{1}$ indicates the cylindrical aperture in which $Y_{c y l}$ is measured and $R_{b}$ denotes the radial extent of the cluster. We assume $R_{b}=10 R_{500}[99], R_{1}=0.75^{\prime} R_{500}$ corresponding to the SPT measurement, and $R_{2}=R_{500}$, since $Y_{X}$ measurements are provided at $R_{500}$. Note that in [101], the SPT collaboration assumed $R_{b}=5 R_{500}$, in order to convert $Y_{S Z}(c y l)$ to $Y_{S Z}(s p h)$, for a sample of low-mass clusters and groups. However, the difference in 


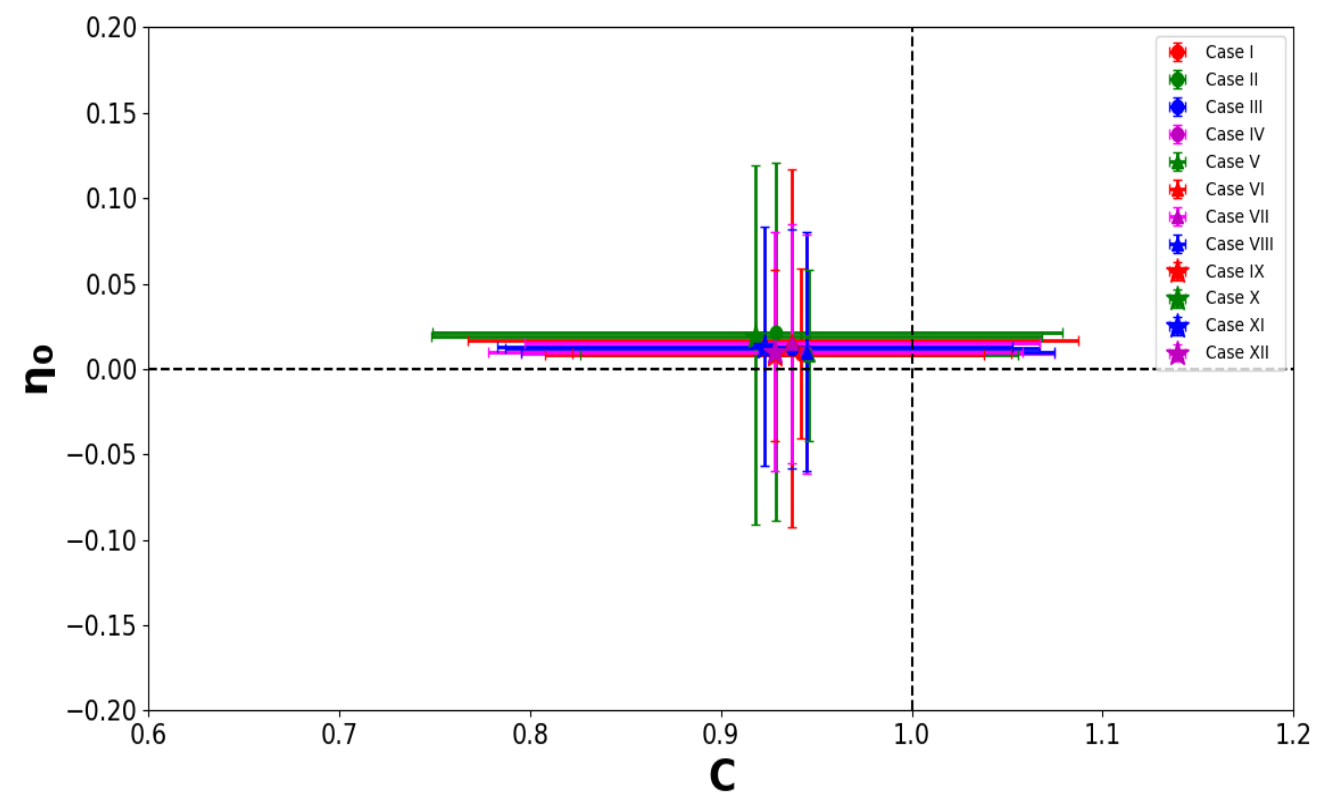

FIG. 8: A comparison of different cases studied in this work. The black dashed lines $\left(C, \eta_{0}\right)=(1,0)$ correspond to the standard distance duality relation. A combination of different colors with different data style represent all different cases used in this study. For all the analyses carried out, $\eta_{0}$ is consistent with zero.

the estimation of $Y_{S Z}(s p h)$ with these two values of $R_{b}$ is negligible. In Eq. 31, $P(r)$ refers to the pressure profile. To do the conversion, we have used the Universal Pressure Profile (UPP) for $P(r)$ [99], similar to our previous work [64].

$$
\frac{P(r)}{P_{500}}=\frac{P_{0}}{x^{\gamma}\left(1+x^{\alpha}\right)^{(\beta-\gamma) / \alpha}}
$$

where $x=r / R_{500}$, and $P_{500}$ is given by [102]

$P_{500}=1.65 \times 10^{-3} E(z)^{8 / 3}\left[\frac{M_{500}}{3 \times 10^{14} h_{70}^{-1} M_{\odot}}\right]^{2 / 3} h_{70}^{2} k e V c m^{-3}$

As a cross-check we also did the conversion using the Battaglia et al (BPP) pressure profile [103], which is given by

$$
P / P_{500}=A\left[1+\left(x / x_{c}\right)^{\alpha}\right]^{-\gamma / \alpha}
$$

where $x=r / R_{500}$; and the values of the constants $A, \alpha$, and $\gamma$ are provided in [103]. A histogram of the difference in $Y_{S Z}(s p h)$ between the two pressure profiles is shown in Fig. 9. Since the difference between the two estimates is negligible, we do only report results using the UPP profile in this manuscript.

Therefore, from Eq. 30 and Eq. 31, we can estimate the ratio of $Y_{s p h}\left(R_{500}\right)$ to $Y_{c y l}\left(0.75^{\prime} D_{A}\right)$. By using this ratio one can estimate $Y_{s p h}\left(R_{500}\right)$ for every SPT-SZ cluster.

\section{ACKNOWLEDGEMENT}

[1] I. M. H. Etherington, Philosophical Magazine 15, 761 (1933).

[2] G. F. R. Ellis, General Relativity and Gravitation 39, 1047 (2007).

[3] B. A. Bassett and M. Kunz, Phys. Rev. D 69, 101305 (2004), URL https://link.aps.org/doi/10. 1103/PhysRevD.69.101305.

[4] J. Qin, F. Melia, and T.-J. Zhang, Mon. Not. R. Astron.
Soc. 502, 3500 (2021), 2101.05574.

[5] B. Ratra and M. S. Vogeley, PASP 120, 235 (2008), 0706.1565.

[6] Planck Collaboration, N. Aghanim, Y. Akrami, M. Ashdown, J. Aumont, C. Baccigalupi, M. Ballardini, A. J. Banday, R. B. Barreiro, N. Bartolo, et al., Astron. \& Astrophys. 641, A6 (2020), 1807.06209.

[7] E. Di Valentino, O. Mena, S. Pan, L. Visinelli, W. Yang, 


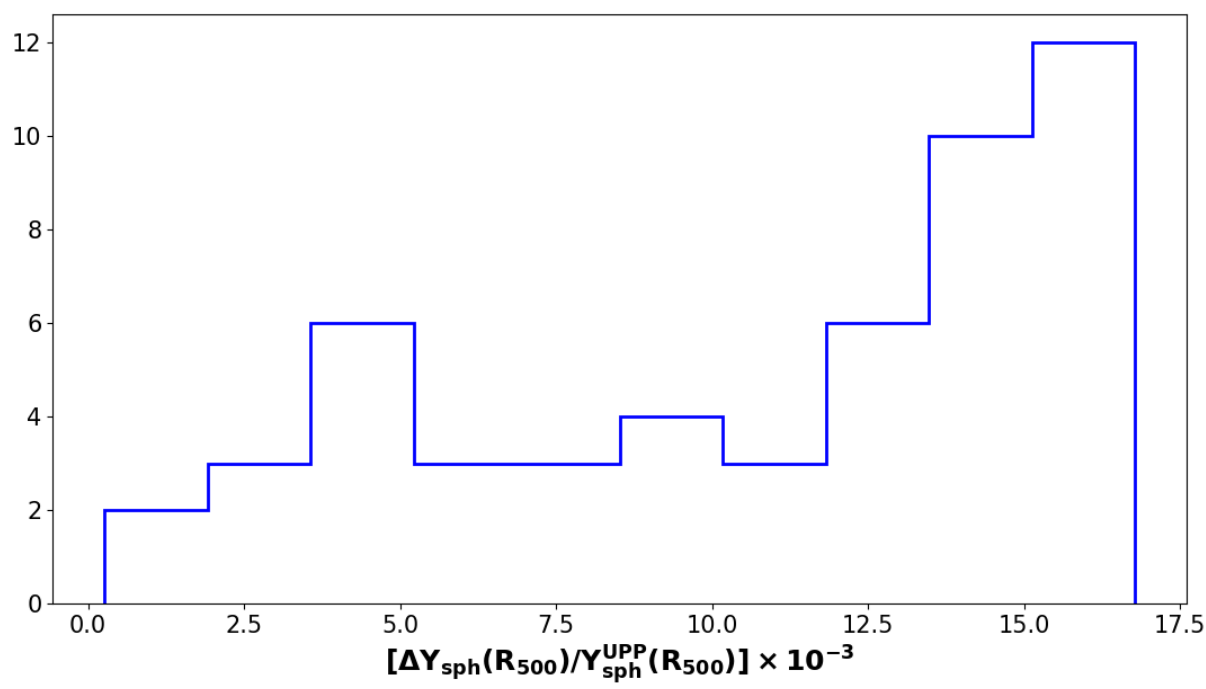

FIG. 9: Histogram of the fractional deviation in $Y_{s p h}\left(R_{500}\right)$ between the UPP and BPP pressure profile for the SPT-SZ clusters analyzed in this work. As we can see, the fractional deviation is less than $1 \%$.

A. Melchiorri, D. F. Mota, A. G. Riess, and J. Silk, arXiv e-prints arXiv:2103.01183 (2021), 2103.01183.

[8] L. Verde, T. Treu, and A. G. Riess, Nature Astronomy 3, 891 (2019), 1907.10625.

[9] S. Bethapudi and S. Desai, Eur. Phys. J. Plus 132, 78 (2017), 1701.01789.

[10] D. Benisty, Physics of the Dark Universe 31, 100766 (2021), 2005.03751.

[11] B. D. Fields, K. A. Olive, T.-H. Yeh, and C. Young, JCAP 2020, 010 (2020), 1912.01132.

[12] J. S. Bullock and M. Boylan-Kolchin, Ann. Rev. Astron. Astrophys. 55, 343 (2017), 1707.04256.

[13] D. H. Weinberg, J. S. Bullock, F. Governato, R. Kuzio de Naray, and A. H. G. Peter, Proceedings of the National Academy of Science 112, 12249 (2015), 1306.0913.

[14] D. Merritt, Studies in the History and Philosophy of Modern Physics 57, 41 (2017), 1703.02389.

[15] S. S. McGaugh, F. Lelli, and J. M. Schombert, Phys. Rev. Lett. 117, 201101 (2016), 1609.05917.

[16] S. Pradyumna, S. Gupta, S. Seeram, and S. Desai, Physics of the Dark Universe 31, 100765 (2021), 2011.06421.

[17] J.-P. Uzan, N. Aghanim, and Y. Mellier, Phys. Rev. D 70, 083533 (2004), URL https://link.aps.org/doi/ 10.1103/PhysRevD .70.083533.

[18] F. de Bernardis, E. Giusarma, and A. Melchiorri, International Journal of Modern Physics D 15, 759 (2006), gr-qc/0606029.

[19] S. Khedekar and S. Chakraborti, Phys. Rev. Lett. 106, 221301 (2011), URL https://link.aps.org/doi/10. 1103/PhysRevLett.106.221301.

[20] Z. Li, P. Wu, and H. Yu, Astrophys. J. Lett. 729, L14 (2011), 1101.5255.

[21] R. Nair, S. Jhingan, and D. Jain, JCAP 2011, 023 (2011), 1102.1065.

[22] X.-L. Meng, T.-J. Zhang, H. Zhan, and X. Wang, As- trophys. J. 745, 98 (2012), 1104.2833.

[23] G. F. R. Ellis, R. Poltis, J.-P. Uzan, and A. Weltman, Phys. Rev. D 87, 103530 (2013), URL https://link. aps.org/doi/10.1103/PhysRevD .87.103530.

[24] K. Liao, Z. Li, S. Cao, M. Biesiada, X. Zheng, and Z.-H. Zhu, Astrophys. J. 822, 74 (2016), 1511.01318.

[25] M.-Z. Lv and J.-Q. Xia, Physics of the Dark Universe 13, 139 (2016), 1606.08102.

[26] X. Li and H.-N. Lin, Mon. Not. R. Astron. Soc. 474, 313 (2018), 1710.11361.

[27] H.-N. Lin, M.-H. Li, and X. Li, Mon. Not. R. Astron. Soc. 480, 3117 (2018), 1808.01784.

[28] T. Yang, R. F. L. Holanda, and B. Hu, Astroparticle Physics 108, 57 (2019).

[29] C.-Z. Ruan, F. Melia, and T.-J. Zhang, Astrophys. J. 866, 31 (2018), 1808.09331.

[30] M.-Z. Lyu, Z.-X. Li, and J.-Q. Xia, Astrophys. J. 888, $32(2020)$.

[31] R. Arjona, JCAP 2020, 009 (2020), 2002.12700.

[32] R. F. L. Holanda, L. R. Colaço, S. H. Pereira, and R. Silva, JCAP 2019, 008 (2019), 1904.01342.

[33] S. W. Allen, A. E. Evrard, and A. B. Mantz, Ann. Rev. Astron. Astrophys. 49, 409 (2011), 1103.4829.

[34] A. V. Kravtsov and S. Borgani, Ann. Rev. Astron. Astrophys. 50, 353 (2012), 1205.5556.

[35] A. A. Vikhlinin, A. V. Kravtsov, M. L. Markevich, R. A. Sunyaev, and E. M. Churazov, Physics Uspekhi 57, 317341 (2014).

[36] S. Desai, Physics Letters B 778, 325 (2018), 1708.06502.

[37] E. D. Reese, J. E. Carlstrom, M. Joy, J. J. Mohr, L. Grego, and W. L. Holzapfel, Astrophys. J. 581, 53 (2002), astro-ph/0205350.

[38] R. F. L. Holanda, J. A. S. Lima, and M. B. Ribeiro, Astrophys. J. Lett. 722, L233 (2010), 1005.4458.

[39] M. Hicken, W. M. Wood-Vasey, S. Blondin, P. Challis, S. Jha, P. L. Kelly, A. Rest, and R. P. Kirshner, Astrophys. J. 700, 1097 (2009), 0901.4804. 
[40] S. J. LaRoque, M. Bonamente, J. E. Carlstrom, M. K. Joy, D. Nagai, E. D. Reese, and K. S. Dawson, Astrophys. J. 652, 917 (2006), astro-ph/0604039.

[41] S. Ettori, A. Morandi, P. Tozzi, I. Balestra, S. Borgani, P. Rosati, L. Lovisari, and F. Terenziani, Astron. \& Astrophys. 501, 61 (2009), 0904.2740.

[42] R. S. Gonçalves, R. F. L. Holanda, and J. S. Alcaniz, Mon. Not. R. Astron. Soc. 420, L43 (2012), 1109.2790.

[43] R. Amanullah, C. Lidman, D. Rubin, G. Aldering, P. Astier, K. Barbary, M. S. Burns, A. Conley, K. S. Dawson, S. E. Deustua, et al., Astrophys. J. 716, 712 (2010), 1004.1711.

[44] R. F. L. Holanda, R. S. Gonçalves, and J. S. Alcaniz, JCAP 2012, 022 (2012), 1201.2378.

[45] N. Liang, Z. Li, P. Wu, S. Cao, K. Liao, and Z.-H. Zhu, Mon. Not. R. Astron. Soc. 436, 1017 (2013), 1104.2497.

[46] M. Bonamente, M. K. Joy, S. J. LaRoque, J. E. Carlstrom, E. D. Reese, and K. S. Dawson, Astrophys. J. 647, 25 (2006), astro-ph/0512349.

[47] X. Yang, H.-R. Yu, Z.-S. Zhang, and T.-J. Zhang, Astrophys. J. Lett. 777, L24 (2013), 1310.0869.

[48] E. De Filippis, M. Sereno, M. W. Bautz, and G. Longo, Astrophys. J. 625, 108 (2005), astro-ph/0502153.

[49] S. Santos-da-Costa, V. C. Busti, and R. F. L. Holanda, JCAP 2015, 061 (2015), 1506.00145.

[50] R. F. L. Holanda and S. H. Pereira, Phys. Rev. D 94, 104037 (2016), 1610.01512.

[51] N. Suzuki, D. Rubin, C. Lidman, G. Aldering, R. Amanullah, K. Barbary, L. F. Barrientos, J. Botyanszki, M. Brodwin, N. Connolly, et al., Astrophys. J. 746, 85 (2012), 1105.3470.

[52] G. Luzzi, M. Shimon, L. Lamagna, Y. Rephaeli, M. De Petris, A. Conte, S. De Gregori, and E. S. Battistelli, Astrophys. J. 705, 1122 (2009), 0909.2815.

[53] G. Hurier, N. Aghanim, M. Douspis, and E. Pointecouteau, Astron. \& Astrophys. 561, A143 (2014), 1311.4694 .

[54] R. F. L. Holanda, V. C. Busti, F. S. Lima, and J. S. Alcaniz, JCAP 2017, 039 (2017), 1611.09426.

[55] R. F. L. Holanda, S. H. Pereira, V. C. Busti, and C. H. G. Bessa, Classical and Quantum Gravity 34, 195003 (2017), 1705.05439.

[56] Planck Collaboration, P. A. R. Ade, N. Aghanim, M. Arnaud, M. Ashdown, J. Aumont, C. Baccigalupi, A. Balbi, A. J. Banday, R. B. Barreiro, et al., Astron. \& Astrophys. 536, A11 (2011), 1101.2026.

[57] D. M. Scolnic, D. O. Jones, A. Rest, Y. C. Pan, R. Chornock, R. J. Foley, M. E. Huber, R. Kessler, G. Narayan, A. G. Riess, et al., Astrophys. J. 859, 101 (2018), 1710.00845.

[58] W. J. C. da Silva, R. F. L. Holanda, and R. Silva, Phys. Rev. D 102, 063513 (2020), 2005.04131.

[59] A. B. Mantz, S. W. Allen, R. G. Morris, D. A. Rapetti, D. E. Applegate, P. L. Kelly, A. von der Linden, and R. W. Schmidt, Mon. Not. R. Astron. Soc. 440, 2077 (2014), 1402.6212.

[60] R. A. Sunyaev and Y. B. Zeldovich, Comments on Astrophysics and Space Physics 4, 173 (1972).

[61] M. Birkinshaw, Physics Reports 310, 97 (1999), astro$\mathrm{ph} / 9808050$.

[62] J. E. Carlstrom, G. P. Holder, and E. D. Reese, Ann. Rev. Astron. Astrophys. 40, 643 (2002), astro$\mathrm{ph} / 0208192$.

[63] T. Mroczkowski, D. Nagai, K. Basu, J. Chluba, J. Say- ers, R. Adam, E. Churazov, A. Crites, L. Di Mascolo, D. Eckert, et al., Space Science Reviews 215, 17 (2019), 1811.02310.

[64] K. Bora and S. Desai, JCAP 2021, 012 (2021), 2008.10541.

[65] C. L. Sarazin, Reviews of Modern Physics 58, 1 (1986).

[66] A. V. Kravtsov, A. Vikhlinin, and D. Nagai, Astrophys. J. 650, 128 (2006), astro-ph/0603205.

[67] S. Sasaki, PASJ 48, L119 (1996), astro-ph/9611033.

[68] L. R. Colaço, R. F. L. Holanda, R. Silva, and J. S. Alcaniz, JCAP 2019, 014 (2019), 1901.10947.

[69] A. Hees, O. Minazzoli, and J. Larena, Phys. Rev. D 90, 124064 (2014), 1406.6187.

[70] O. Minazzoli and A. Hees, Phys. Rev. D 90, 023017 (2014), 1404.4266

[71] R. S. Gonçalves, S. Landau, J. S. Alcaniz, and R. F. L. Holanda, JCAP 2020, 036 (2020), 1907.02118.

[72] E. Rozo, A. Vikhlinin, and S. More, Astrophys. J. 760, 67 (2012), 1202.2150

[73] S. Planelles, D. Fabjan, S. Borgani, G. Murante, E. Rasia, V. Biffi, N. Truong, C. Ragone-Figueroa, G. L. Granato, K. Dolag, et al., Mon. Not. R. Astron. Soc. 467, 3827 (2017), 1612.07260.

[74] V. Biffi, F. Sembolini, M. De Petris, R. Valdarnini, G. Yepes, and S. Gottlöber, Mon. Not. R. Astron. Soc. 439, 588 (2014), 1401.2992.

[75] D. Fabjan, S. Borgani, E. Rasia, A. Bonafede, K. Dolag, G. Murante, and L. Tornatore, Mon. Not. R. Astron. Soc. 416, 801 (2011), 1102.2903.

[76] S. T. Kay, M. W. Peel, C. J. Short, P. A. Thomas, O. E. Young, R. A. Battye, A. R. Liddle, and F. R. Pearce, Mon. Not. R. Astron. Soc. 422, 1999 (2012), 1112.3769.

[77] R. Stanek, E. Rasia, A. E. Evrard, F. Pearce, and L. Gazzola, Astrophys. J. 715, 1508 (2010), 0910.1599.

[78] C. Loken, M. L. Norman, E. Nelson, J. Burns, G. L. Bryan, and P. Motl, Astrophys. J. 579, 571 (2002), astro-ph/0207095.

[79] S. Galli, Phys. Rev. D 87, 123516 (2013), 1212.1075.

[80] E. Bulbul, I. N. Chiu, J. J. Mohr, M. McDonald, B. Benson, M. W. Bautz, M. Bayliss, L. Bleem, M. Brodwin, S. Bocquet, et al., Astrophys. J. 871, 50 (2019), 1807.02556.

[81] J. E. Carlstrom, P. A. R. Ade, K. A. Aird, B. A. Benson, L. E. Bleem, S. Busetti, C. L. Chang, E. Chauvin, H. M. Cho, T. M. Crawford, et al., PASP 123, 568 (2011), 0907.4445 .

[82] L. E. Bleem, B. Stalder, T. de Haan, K. A. Aird, S. W. Allen, D. E. Applegate, M. L. N. Ashby, M. Bautz, M. Bayliss, B. A. Benson, et al., Astrophys. J. Suppl. Ser. 216, 27 (2015), 1409.0850.

[83] S. Bocquet, J. P. Dietrich, T. Schrabback, L. E. Bleem, M. Klein, S. W. Allen, D. E. Applegate, M. L. N. Ashby, M. Bautz, M. Bayliss, et al., Astrophys. J. 878, 55 (2019), 1812.01679.

[84] A. Saro, S. Bocquet, E. Rozo, B. A. Benson, J. Mohr, E. S. Rykoff, M. Soares-Santos, L. Bleem, S. Dodelson, P. Melchior, et al., Mon. Not. R. Astron. Soc. 454, 2305 (2015), 1506.07814.

[85] S. Desai, R. Armstrong, J. J. Mohr, D. R. Semler, J. Liu, E. Bertin, S. S. Allam, W. A. Barkhouse, G. Bazin, E. J. Buckley-Geer, et al., Astrophys. J. 757, 83 (2012), 1204.1210.

[86] J. Song, A. Zenteno, B. Stalder, S. Desai, L. E. Bleem, K. A. Aird, R. Armstrong, M. L. N. Ashby, 
M. Bayliss, G. Bazin, et al., Astrophys. J. 761, 22 (2012), 1207.4369.

[87] D. Huterer and D. L. Shafer, Reports on Progress in Physics 81, 016901 (2018), 1709.01091.

[88] P. Wu, Z. Li, X. Liu, and H. Yu, Phys. Rev. D 92, 023520 (2015).

[89] A. G. Riess, S. Casertano, W. Yuan, J. B. Bowers, L. Macri, J. C. Zinn, and D. Scolnic, Astrophys. J. Lett. 908, L6 (2021), 2012.08534.

[90] M. Seikel, C. Clarkson, and M. Smith, JCAP 2012, 036 (2012), 1204.2832.

[91] H. Singirikonda and S. Desai, European Physical Journal C 80, 694 (2020), 2003.00494.

[92] K. Bora and S. Desai, European Physical Journal C 81, 296 (2021), 2103.12695.

[93] F. Pedregosa, G. Varoquaux, A. Gramfort, V. Michel, B. Thirion, O. Grisel, M. Blondel, P. Prettenhofer, R. Weiss, V. Dubourg, et al., Journal of Machine Learning Research 12, 2825 (2011).

[94] E.-K. Li, M. Du, Z.-H. Zhou, H. Zhang, and L. Xu, Mon. Not. R. Astron. Soc. 501, 4452 (2021), 1911.12076.

[95] R. Jimenez and A. Loeb, Astrophys. J. 573, 37 (2002), astro-ph/0106145.
[96] D. Foreman-Mackey, D. W. Hogg, D. Lang, and J. Goodman, PASP 125, 306 (2013), 1202.3665.

[97] X.-Y. Fu, P.-X. Wu, H.-W. Yu, and Z.-X. Li, Research in Astronomy and Astrophysics 11, 895 (2011).

[98] D. Foreman-Mackey, The Journal of Open Source Software 1, 24 (2016), URL https://doi.org/10.21105/ joss. 00024.

[99] M. Arnaud, G. W. Pratt, R. Piffaretti, H. Böhringer, J. H. Croston, and E. Pointecouteau, Astron. \& Astrophys. 517, A92 (2010), 0910.1234.

[100] J. B. Melin, J. G. Bartlett, J. Delabrouille, M. Arnaud, R. Piffaretti, and G. W. Pratt, Astron. \& Astrophys. 525, A139 (2011), 1001.0871.

[101] J. Liu, J. Mohr, A. Saro, K. A. Aird, M. L. N. Ashby, M. Bautz, M. Bayliss, B. A. Benson, L. E. Bleem, S. Bocquet, et al., Mon. Not. R. Astron. Soc. 448, 2085 (2015), 1407.7520.

[102] D. Nagai, A. V. Kravtsov, and A. Vikhlinin, Astrophys. J. 668, 1 (2007), astro-ph/0703661.

[103] N. Battaglia, J. R. Bond, C. Pfrommer, J. L. Sievers, and D. Sijacki, Astrophys. J. 725, 91 (2010), 1003.4256. 